\title{
Pre-Whitened Dithered Signed-Error Constant Modulus Algorithm for Efficient Blind Channel Equalization
}

\author{
Naveed R. Butt and L. Cheded
}

\begin{abstract}
Blind channel equalization has gained great importance in the world of communications. Among a large number of available blind equalization algorithms, the CMA (Constant Modulus Algorithm) enjoys widespread popularity because of its LMS-like complexity and robustness. Two important improvements on the CMA performance are the Dithered Signed-Error CMA (DSE-CMA) and the PreWhitened CMA (PW-CMA). The DSE-CMA is an approach to reduce the computational complexity of the CMA while retaining its robustness and the $\mathrm{PW}-\mathrm{CMA}$ aims at improving the convergence rate of the CMA in case of channels exhibiting large frequency response deviations.

In this paper we review the two approaches and propose a new scheme combining the virtues of the two. The combined scheme is computationally simpler than the PW-CMA and provides better convergence than the DSE-CMA. It is particularly suited for the situations where ill-convergence needs to be treated with minimum additional complexity and without loss of robustness.
\end{abstract}

\section{INTRODUCTION}

$\mathrm{I}^{\mathrm{r}}$ NFORMATION bearing signals transmitted between remote locations often encounter a signal-altering physical channel. Examples of common physical channels include coaxial, fiber optic, or twisted-pair cable in wired communications and the atmosphere or ocean in wireless communications. These physical channels cause distortions in the signal, thereby rendering it partially or completely meaningless. Channel equalization, an approach commonly used to counter the effects of channel distortion, can be viewed as the application of a linear filter (called the equalizer) to the received signal. The equalizer attempts to extract the transmitted symbol sequence by counteracting the effects of the channel, consequently improving the probability of correct symbol detection.

Since it is common for the channel characteristics to be unknown (e.g., at startup) or to change over time, the preferred embodiment of the equalizer is a structure adaptive in nature. Classical equalization techniques employ a timeslot (recurring periodically for time-varying situations) during which a training signal, known in advance by the

This work was supported by the King Fahd University of Petroleum and Minerals, Dhahran, Saudi Arabia.

N. R. Butt and L. Cheded are with the Systems Engineering Department, College of Computer Science and Engineering at the King Fahd University of Petroleum and Minerals, Dhahran, Saudi Arabia.

Contacts: nrbutt@ccse.kfupm.edu.sa, cheded@ccse.kfupm.edu.sa receiver, is transmitted. The receiver adapts the equalizer so that its output closely matches the known reference training signal [1]. Since the inclusion of such signals sacrifices valuable channel capacity, adaptation without resort to training, i.e., blind adaptation, is preferred and has become a topic of great interest. Blind equalization [2] refers to the adaptive equalization of physical communication channels without the need of any training sequence. The Constant Modulus Algorithm (CMA) [3]-[5] is a commonly used blind channel equalization algorithm that relies on the constant modulus property of a particular class of signals (e.g. BPSK and QPSK).

In spite of its robustness [5], the CMA suffers form two main limitations i) It requires a rather large number of update multiplications (computational complexity) [4], ii) it exhibits extremely slow convergence in case of channels offering high frequency deviations [6]. The Signed Error CMA (SE-CMA) is an approach to replace the bulk of CMA multiplications by simple sign operations [4]. This approach works well under favorable conditions; however it has been shown to lack robustness (ability to perform well under violation of the Perfect Equalizability Conditions) [7]. To improve the robustness of the SE-CMA, a new approach called the Dithered SE-CMA (DSE-CMA) has been proposed in [8]. The DSE-CMA treats the SE-CMA as a one-bit quantizer and makes judicious use of a controlled random noise (the Dither) to make the quantization noise independent of the signal being quantized. This, in effect, adds robustness to the SE-CMA.

To overcome the second limitation, [6] have proposed the use of blind adaptive pre-whitening filter in the equalization process. This is called the Pre-whitened CMA (PW-CMA). The attempt here is to reduce the eigenvalue spread of the received signal before sending it to the equalizer. This results in improved convergence behavior. The approach is particularly useful in case of channels offering high frequency deviations and outperforms its common counterpart the CMA.

Each of the two approaches mentioned above performs well in one area but not in the other. For example, the DSECMA is computationally less expensive than the CMA; however, as shown in the present study, it fails to provide good convergence in case of channels with high frequency deviations. On the other hand the PW-CMA that provides better convergence behavior uses the computationally expensive constant modulus algorithm. It is therefore of practical interest to devise algorithms that can perform well 
in both respects. This paper proposes to use the DSE-CMA with the pre-whitening filter to obtain a scheme that is computationally less expensive than the PW-CMA and provides better convergence than the CMA and the DSECMA.

The rest of the work has been organized as follows. Section II provides an introductory framework that will be helpful in later sections. Section III is dedicated to a review of the various improvements on the CMA, while section IV presents the new proposed scheme the PW-DSE-CMA. Simulation results that verify the effectiveness of the proposed scheme are also included in section IV.

Some notational details are as follows: lower-case bold face letters are used to denote vectors, while upper-case bold face letters represent matrices. The notations (.) , (.) $)^{*}\lfloor$ represent transpose, complex conjugate and the floor function, respectively.

\section{PRELIMINARIES}

In this section we present introductory framework that will be helpful in the later sections. In particular, we provide a brief development of the equalization model and the CMA. This is followed by a review of the notions of Perfect Blind Equalizability and Robustness. Finally, the convergence indices and channels used in this study are presented.

\section{A. The Equalization Model}

To introduce the equalization model we make use of the following block diagram.

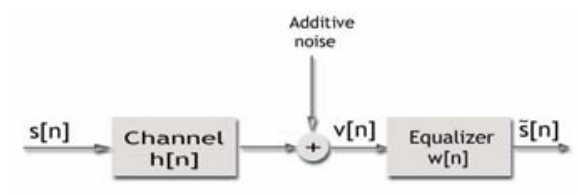

Figure 1: The Channel and Equalizer

Here $\{s[n]\}$ is the transmitted sequence which is assumed to come from a finite, zero mean symmetric alphabet. The transmission channel is represented by its impulse response (IR) coefficients $\{h[n]\}$, and $\{\eta[n]\}$ represents the additive white Gaussian noise. The received sequence is thus given by

$$
v[n]=\sum_{k=0}^{N h} h[k] s[n-k]+\eta[n]
$$

Here $N h$ represents the channel IR length. Assuming that the equalizer IR has length $N w$ and defining $N q=\lfloor(N h+N w-1) / 2\rfloor$, we can define a vector $\mathbf{v}[n]$ containing last $N w$ received symbols, a vector $\mathbf{s}[n]$ containing last $N q$ transmitted symbols and a vector $\boldsymbol{\eta}[n]$ containing last $N w$ samples of the additive noise. These can be related through the $N w \quad x \quad N q$ fractionally spaced convolution matrix $\mathbf{H}$ as [8]:

$$
\mathbf{v}[n]=\mathbf{H s}[n]+\boldsymbol{\eta}[n]
$$

Where

$$
\mathbf{H}=\left[\begin{array}{ccccc}
h[1] & h[3] & \ldots & h[N h-1] & \\
h[0] & h[2] & \ldots & h[N h-2] & \\
& h[1] & h[3] & \ldots & h[N h-1] \\
& h[0] & h[2] & \ldots & h[N h-2] \\
& & . & . & .
\end{array}\right]
$$

Finally the equalizer output symbol at instant $n$ can be written in terms of the $N w$-length equalizer coefficient vector $\boldsymbol{w}$ as:

$$
\tilde{S}[n]=\mathbf{w}^{t} \mathbf{H s}[n]+\mathbf{w}^{t} \boldsymbol{\eta}[n]
$$

\section{B. The Constant Modulus Algorithm (CMA)}

The CMA is a stochastic gradient algorithm that assumes that all the transmitted symbols have equal modulus value and moves the output of the equalizer towards that value. It is a special case of the so-called Godard algorithm [3, 4] which minimizes a cost function

$$
J_{C M}[n]=\frac{1}{4} E\left[\left(R_{2}-|\tilde{s}[n]|^{2}\right)^{2}\right]
$$

where, $R_{2}$ represents the modulus of the transmitted symbols which is considered by CMA as being constant i.e.

$$
R_{2}=|s[n]|^{2}=\text { constant }
$$

Note that the CMA cost function penalizes the equalizer outputs that do not have a constant modulus (equaling $R_{2}$ ). An update equation for the weights of the CMA equalizer takes the following form ( $\mu$ is a small positive step size):

$$
\hat{\mathbf{w}}(n+1)=\hat{\mathbf{w}}(n)+\mu \mathbf{v}^{*}[n]\left(R_{2}-|\tilde{s}[n]|^{2}\right) \tilde{s}[n]
$$

Its error function is given as

$$
\psi(\tilde{s}[n])=\left(R_{2}-|\tilde{s}[n]|^{2}\right) \tilde{s}[n]
$$

The CMA is well known to possess superior robustness properties. A detailed analysis of the CMA properties can be found in [5]. 


\section{Perfect Blind Equalizability and Robustness}

In the context of channel equalization, robustness of an equalizer refers to its ability to perform well under the violation of a set of conditions called the Perfect Blind Equalizability (PBE) conditions. These have been shown [5] to guarantee perfect symbol recovery for algorithms minimizing cost function given in (4). These conditions are:

P1) Full Column-rank $\mathbf{H}$

P2) No additive channel noise

P3) Sub-Gaussian source

P4) Zero mean, i.i.d. source

\section{Convergence Indices: MSE and ISI}

We shall make use of the following two parameters to study the convergence behavior of the CMA and its variants.

MSE: The Mean Square Error (MSE) is taken as the square of the difference between the modulus of the equalizer output and the constant modulus $R_{2}$ (for BPSK $R_{2}$ $=1$ ). It is usually converted to dB's.

$$
\begin{aligned}
& \operatorname{MSE}=\left(R_{2}-|\tilde{S}[n]|^{2}\right)^{2} \\
& \operatorname{MSE}(d B)=20 \log _{10}|M S E|
\end{aligned}
$$

ISI: If the overall IR from the transmitted symbol to the equalizer output is given by $\{I[n]\}$ then The Inter-SymbolInterference (ISI) measure is defined as

$$
\begin{aligned}
& I S I=\frac{\sum_{n} I[n]^{2}-\max _{n}\left(I[n]^{2}\right)}{\max _{n}\left(I[n]^{2}\right)} \\
& I S I(d B)=20 \log _{10}|I S I|
\end{aligned}
$$

\section{E. Channels Used}

We make use of the following two channels to study the different schemes. These channels have been selected to properly elucidate the characteristics of the schemes under study.

Channel $1: \mathrm{h}=(0.1,0.3,1,-0.1,0.5,0.2)$, this channel is selected to represent situations where we do not have high frequency response deviations. We shall conduct simulations of this channel with and without violations of the PBE conditions.

Channel 2: $\mathrm{h}=(.0003, .0029, .0090,-.0440, .0468$, $-0.485, .3603,-.7401, .5451,-.1326, .0350)$. Adapted from [6], this channel has been selected to exhibit high frequency response deviations. It is well known that CMA exhibits illconvergence in such cases.

\section{SignED-ERROR, DitHERED SigNED-ERROR AND PRE- WHITENED CMA}

\section{A. Signed-Error CMA}

Signed-Error CMA (SE-CMA) is an attempt to replace the bulk of CMA update multiplications by simple sign operations. This is achieved by redefining the error function and the update equation as (for the present work we restrict our attention to real-valued quantities, extension to complexvalued cases can be done on the lines of [9])

$$
\begin{gathered}
\xi(\tilde{s}[n])=\operatorname{sgn}\left(\left(R_{2}-|\tilde{s}[n]|^{2}\right) \tilde{s}[n]\right) \\
\hat{\mathbf{w}}(n+1)=\hat{\mathbf{w}}(n)+\mu \mathbf{v}[n] \operatorname{sgn}\left(\left(R_{2}-|\tilde{s}[n]|^{2}\right) \tilde{s}[n]\right)
\end{gathered}
$$

Thus only the sign of the error is retained [10]. The SECMA is very efficient in reducing the computational complexity of the CMA. However, recent investigations suggest that the SE-CMA lacks robustness [7]. Thus violations in the PBE conditions can severely hinder SECMA convergence behavior.

\section{B. Dithered Signed-Error CMA}

In an attempt to find computationally efficient algorithms that retain the robustness of the CMA, [8] introduced the Dithered SE-CMA (DSE-CMA). The main idea behind this approach is based on the observation that by taking only the sign of the CMA error function we are actually quantizing it (with one bit only). This introduces certain quantization noise into the process which in turn affects the robustness of the SE-CMA. Addition of dither (controlled random noise) before quantization can make the quantization noise white, zero mean and independent of the error signal that is being quantized [11]. This white noise is then expected to get averaged out in the process. The DSE-CMA update equation is given as [12]:

$$
\hat{\mathbf{w}}(n+1)=\hat{\mathbf{w}}(n)+\mu \mathbf{v}[n] \alpha \operatorname{sgn}\left(\left(R_{2}-|\tilde{s}[n]|^{2}\right) \tilde{s}[n]+\alpha d_{n}\right)
$$

with error function

$$
\varphi_{\alpha}\left(\tilde{s}[n], d_{n}\right)=\alpha \operatorname{sgn}\left(\left(R_{2}-|\tilde{s}[n]|^{2}\right) \tilde{s}[n]+\alpha d_{n}\right)
$$

Here $d_{n}$ is an i.i.d. dithering process uniformly distributed on $[-1,1]$ and $\alpha$ is a small positive constant.

We present here the effect of $\alpha$ on the transient and steady state behavior of the DSE-CMA (these results are adapted from [8], where interested readers can find detailed analysis of the DSE-CMA characteristics). In transient state the DSE-CMA error function behaves as a "hard-limited" version of the CMA error function, 


$$
\varphi_{\alpha}(\tilde{s})=\left\{\begin{array}{lc}
\alpha & \tilde{s}: \psi(\tilde{s})>\alpha \\
\psi(\tilde{s}) & \tilde{s}:|\psi(\tilde{s})| \leq \alpha \\
\alpha & \tilde{s}: \psi(\tilde{s})<\alpha
\end{array}\right.
$$

In the steady state case the Excess MSE is directly proportional to $\alpha^{2}$. Thus although we can increase $\alpha$ to ensure wider range of CMA-like robustness, we do this at the cost of additional EMSE.

The DSE-CMA has been shown to perform well with proper selection of the parameters. Simulation results for channels 1 and 2 (figs. 5 - 8) show that the DSE-CMA behaves much like the conventional CMA. Thus it reduces the computational complexity while retaining CMA-like robustness. However, the DSE-CMA and the CMA both suffer from slow convergence (particularly in case of channels exhibiting large frequency response deviations). This is evident from figs. $8-11$. This issue will be re-visited in section IV.

\section{Pre-Whitened CMA}

As pointed out in the introduction, the second major problem with the CMA is its slow convergence. This issue has motivated the use of a pre-whitening filter prior to equalization. Following is a brief development of the PWCMA characteristics based on [6].

Consider the following two systems (figs. 2 \& 3),

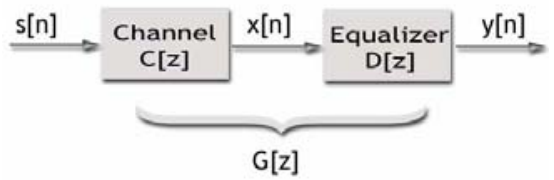

Figure 2: Simple Channel Equalizer Block Diagram

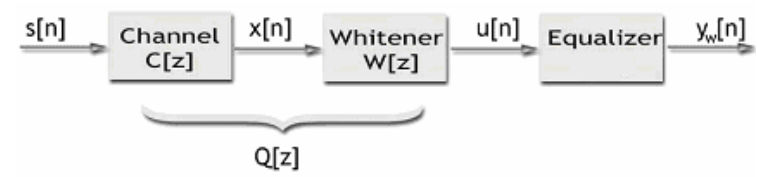

Figure 3: Pre-Whitened Block Diagram

Here again we assume the source sequence $\{s[n]\}$ to be zero mean and i.i.d. Additionally, we assume that the whitener is optimal (thus its output $\{u[n]\}$ is white). The transformation from $\mathbf{s}$ (source sequence vector) to $\mathbf{u}$ (whitener output vector) is given by $\mathbf{u}=\mathbf{Q}^{t} \mathbf{s}$. Here $\mathbf{Q}$ represents the convolution matrix of the cascaded channel-whitener filter $\mathrm{Q}(\mathrm{z})$. We now show that this transformation is unitary. The autocorrelation matrix of the vector $\mathbf{u}$ is given by

$$
\mathbf{R}_{v u}=E\left\{\mathbf{u u ^ { \prime }}\right\}=E\left\{\mathbf{Q}^{\prime} \mathbf{s s}^{\prime} \mathbf{Q}\right\}=\mathbf{Q}^{\prime} E\left\{\mathbf{s s}^{\prime}\right\} \mathbf{Q}=\mathbf{Q}^{\prime} \mathbf{R}_{s s} \mathbf{Q}
$$

Now since both $\mathbf{s}$ and $\mathbf{u}$ are assumed to be white (i.e. $\mathbf{R}_{v w}=\mathbf{R}_{s}=\mathbf{I}$ ), we have:

$$
\mathbf{Q}^{\prime} \mathbf{Q}=\mathbf{I} \text { and } \mathbf{Q}^{\prime}=\mathbf{Q}^{-1}
$$

This shows that transformation from $\mathbf{s}$ to $\mathbf{u}$ is unitary. From functional analysis it is well known that a unitary transformation from one space to another has all eigenvalues equal to one and is isometric. Now the cost functions for the two systems can be given as ( $\mathbf{d}_{\mathbf{w}}$ represents equalizer weights vector for the pre-whitened case)

$$
\begin{aligned}
& J(G)=\frac{1}{4} E\left\{\left(|y(n)|^{2}-R_{z}\right)^{2}\right\} \\
& J\left(\mathbf{d}_{w}\right)=\frac{1}{4} E\left\{\left(\left|y_{v}(n)\right|^{2}-R_{2}\right)^{2}\right\}
\end{aligned}
$$

It has been shown [6] that the gradients of these two functions are related by

$$
\frac{\partial J\left(\mathbf{d}_{w}\right)}{\partial \mathbf{d}}=\mathbf{Q}^{\prime} \frac{\partial J(G)}{\partial G}
$$

The transformation depicted in the above relation is unitary (since $\mathbf{Q}$ is a unitary operator). Such a transformation does not have any effect on the asymptotic behavior but can affect the convergence speed. This clearly suggests that the use of a pre-whitening filter can improve the convergence speed of a CMA equalizer.

An adaptive linear predictor can be used to provide the desired pre-whitening. This is shown in fig. 4

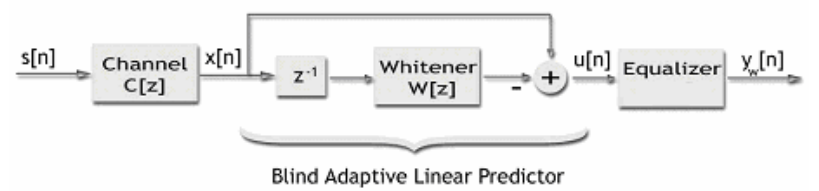

Figure 4: Adaptive Pre-whitening

Note that in this setup it is the error signal (innovation) that is fed to the CMA equalizer. Assuming that the linear predictor has $N$ weights, we can use the LMS adaptation algorithm for the updating of these weights. Defining

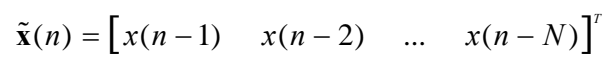

the update equations are 


$$
\begin{aligned}
& u(n)=x(n)-\mathbf{w}^{t}(n) \tilde{\mathbf{x}}(n) \\
& \mathbf{w}(n+1)=\mathbf{w}(n)+\mu_{p} u(n) \tilde{\mathbf{x}}(n)
\end{aligned}
$$

where $\mu_{P}$ represents the adaptation step size for the Prewhitener. Also, assuming that the CMA equalizer has $\mathrm{Nw}$ weights and defining

$$
\mathbf{v}(n)=[u(n) \quad u(n-1) \quad \ldots \quad u(n-(N w-1))]^{T}
$$

the equalizer update equations are

$$
\begin{aligned}
& y_{w}(n)=\mathbf{d}_{w}^{\prime}(n) \mathbf{v}(n) \\
& \mathbf{d}_{w}(n+1)=\mathbf{d}_{w}(n)+\mu_{E} \mathbf{v}(n)\left(R_{2}-\left|y_{w}(n)\right|^{2}\right) y_{w}(n)
\end{aligned}
$$

where $\mu_{E}$ represents the adaptation step size for the CMA Equalizer.

The PW-CMA has been shown to improve the CMA convergence speed significantly (e.g. see figs. 5 - 11). However, this algorithm is computationally more expensive. We say more on this in the next section.

\section{PROPOSED COMBINED SCHEME: THE PRE-WHITENED DITHERED SIGNED-ERROR CMA}

In this section we introduce the proposed scheme; the Prewhitened DSE-CMA (PW-DSE-CMA).

\section{A. Proposed Scheme}

As we saw in the previous sections, the DSE-CMA and the PW-CMA solve the problems of computational complexity and ill-convergence, respectively. However, each deals with only one issue at a time. Thus the DSECMA does not solve the problem of ill-convergence while the PW-CMA introduces computational complexity. In order to have the best of both worlds, we propose to use Prewhitening with the DSE-CMA. This combination is expected to have threefold advantage:

- The PW-DSE-CMA will have better convergence rate than the DSE-CMA.

- The PW-DSE-CMA will have less computational complexity than the PW-CMA.

- The combined scheme will retain the robustness of the CMA (since it is based on the DSE-CMA)

The main idea is to use the blind adaptive linear predictor as shown in fig. 4 with the following adjustments:

- $\quad$ The linear predictor weights are updated using (13).

- The equalization part is performed using the DSECMA (9), instead of the regular CMA. Thus the updated equation in (15) is replaced by:

$$
\mathbf{d}_{w}(n+1)=\mathbf{d}_{w}(n)+\alpha \mu_{E} \mathbf{v}(n) \operatorname{sgn}\left(\left(R_{2}-\left|y_{w}(n)\right|^{2}\right) y_{w}(n)+\alpha d_{n}\right)
$$

Here, $\alpha$ and $d_{n}$ are as defined in section III.B.

\section{B. Simulation Results}

We now present simulation results that demonstrate the effectiveness of the proposed approach and compare it with the existing methods. We used the two channels introduced earlier. The input was PRBS (i.e. $\mathrm{s}[\mathrm{n}]=[+1,-1]$ ) with zero mean and unit variance. In each case we selected $\alpha=0.45$ and standard centre-tap initialization was used. The number of equalizer weights used $(\mathrm{Nw})$ and the SNR are mentioned with each figure. The number of linear predictor weights was set equal to the number of equalizer weights in cases involving pre-whitening. We tested convergence in terms of MSE and ISI. It can be seen from figs. 5 - 9 that in each case the proposed scheme (PW-DSE-CMA) provides better convergence than the DSE-CMA. Additionally, the results show that the proposed scheme can replace its computationally expensive counterpart, the PW-CMA.

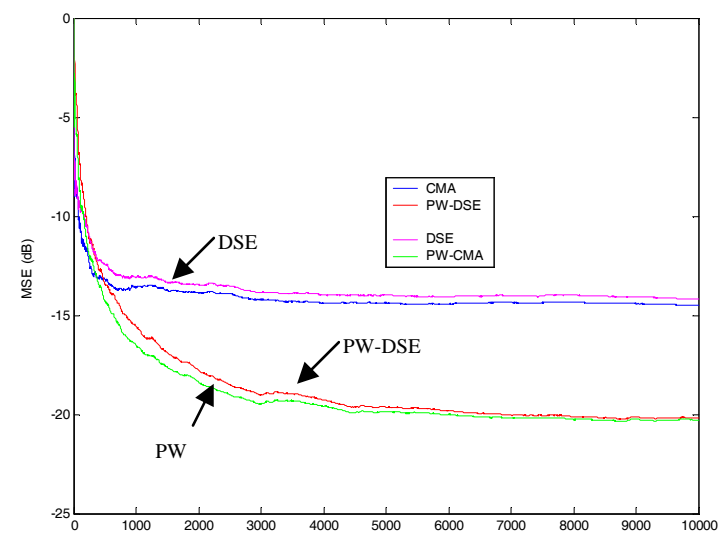

Figure 5: Channel 1, $\mathrm{Nw}=3$, Noiseless, No PBE Violation.

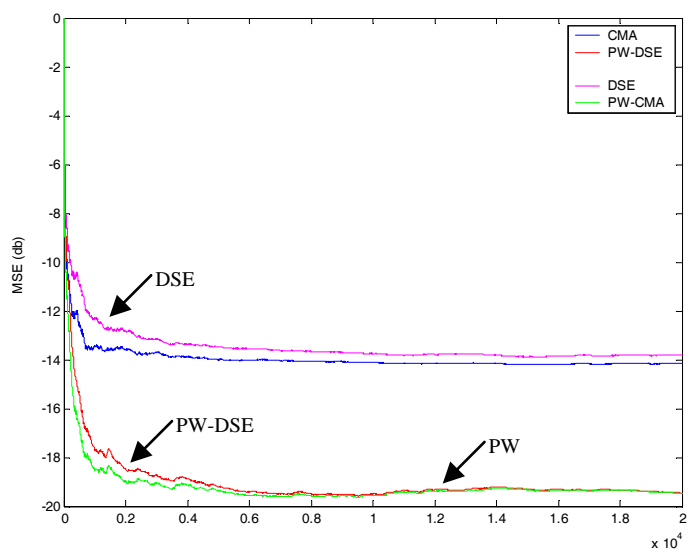


Figure 6: Channel 1, $\mathrm{Nw}=3, \mathrm{SNR}=20 \mathrm{~dB}, \mathrm{P2}$ Violated Mildly.

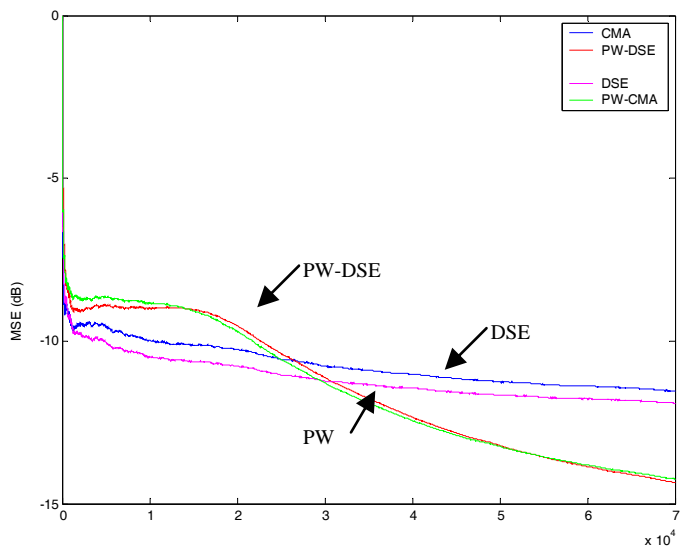

Figure 7: Channel 2, $\mathrm{Nw}=25, \mathrm{SNR}=30 \mathrm{~dB}, \mathrm{P} 1$ and $\mathrm{P2}$ Violated.

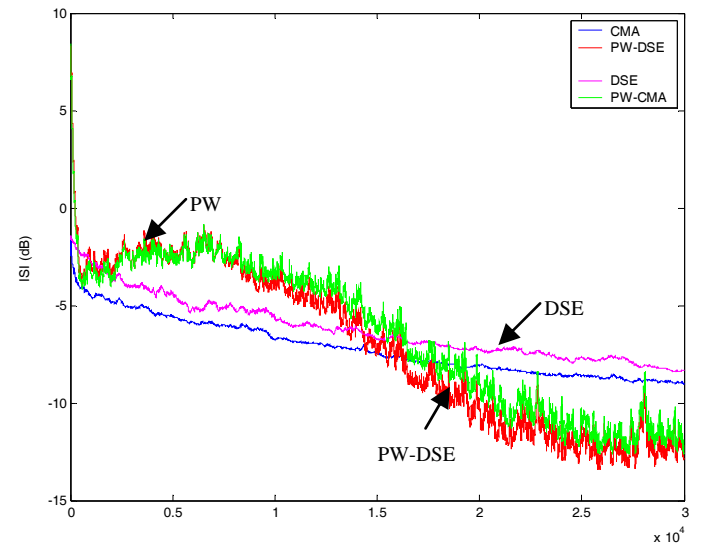

Figure 8: Channel 2, Nw $=25, \mathrm{SNR}=20 \mathrm{~dB}, \mathrm{P1}$ and $\mathrm{P2}$ Violated.

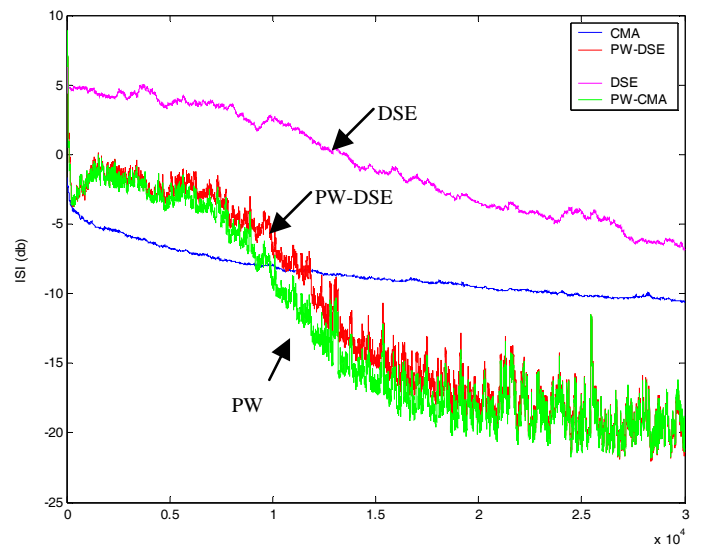

Figure 9: Channel 2, $\mathrm{Nw}=15, \mathrm{SNR}=30 \mathrm{~dB}, \mathrm{P} 1$ and $\mathrm{P2}$ Violated

\section{CONCLUSION}

In this paper a new improved scheme for blind channel equalization is presented. The proposed scheme combines the computationally efficient DSE-CMA with the fast converging PW-CMA to utilize the powers of both. It is shown through simulations that the proposed scheme provides convergence faster than the DSE-CMA while being computationally less expensive than the PW-CMA. Further analysis on the proposed scheme's behavior under more varied situations will be carried out to explore its characteristics.

\section{ACKNOWLEDGMENT}

The authors would like to gratefully acknowledge the support provided by the King Fahd University of Petroleum and Minerals, Dhahran, Saudi Arabia

\section{REFERENCES}

[1] S.U.H. Qureshi, “Adaptive Equalization,” Proc. of the IEEE, vol. 73, no.9, pp. 1349-87, Sept 1985.

[2] S. Haykin, Blind Deconvolution, Englewood Cliff, NJ: Prentice-Hall, 1994.

[3] D.N. Godard, "Self-Recovering Equalization and Carrier Tracking in Two- Dimensional Data Communication Systems," IEEE Trans. on Commun., vol. COM-28, No. 11, pp. 1867-75, Nov. 1980.

[4] J.R. Treichler, M.G. Agee, "A New Approach to Multipath Correction of Constant Modulus Signals," IEEE Trans. on Acoustics, Speech, and Signal Processing, vol. ASSP-31, no. 2 pp. 459-72, April 1983.

[5] C. R. Johnson, Jr., P. Schniter, T.J. Enders, J.D. Behm, D.R. Brown, R.A. Casas, "Blind equalization using the constant modulus criterion: A review," Proceedings of the IEEE special issue on Blind System Identification and Estimation, vol. 86, no. 10, pp. 1987-50, Oct. 1998.

[6] J. P. LeBlanc and I. Fijalkow, "Blind adapted, pre-whitened constant modulus algorithm”, Proc. IEEE Int. Conf. Communications, vol. 8, pp. 2438-2442, Jun. 2001.

[7] D. R. Brown, P. Schniter, and C. R. Johnson, Jr., "Computationally efficient blind equalization," in Proc. 35th Allerton Conf. Commun., Contr., Comput., Monticello, IL, Sept. 1997, pp. 54-63.

[8] P. Schniter and C. R. Johnson, "Dithered Signed-Error CMA: Robust, Computationally Efficient Blind Adaptive Equalization", IEEE Transactions on Signal Processing, vol. 47, No. 6, June 1999.

[9] P. Schniter and C. R. Johnson, Jr., "Dithered signed-error CMA: The complex-valued case," in Proc. Asilomar Conf. Signals, Syst. Comput., Pacific Grove, CA, Nov. 1998.

[10] O. Macchi, Adaptive Processing. New York: Wiley, 1995.

[11] R. M. Gray and T. G. Stockham, Jr., "Dithered quantizers," IEEE Trans.Inform. Theory, vol. 39, pp. 805-812, May 1993.

[12] P. Schniter and C. R. Johnson, Jr., "The dithered signed-error constant modulus algorithm," in Proc. Int. Conf. Acoust., Speech, Signal Process., Seattle, WA, May 1998, pp. 3353-3356. 